\title{
Molecular epidemiological analysis and risk factors for acquisition of carbapenemase- producing Enterobacter cloacae complex in a Japanese university hospital
}

Nobuyuki Tetsuka ${ }^{1,2}$, Aki Hirabayashi $^{3}$, Akane Matsumoto ${ }^{1}$, Keisuke Oka ${ }^{1,2}$, Yuki Hara ${ }^{2,4}$, Hiroshi Morioka ${ }^{1}$, Mitsutaka Iguchi ${ }^{1}$, Yuka Tomita ${ }^{1}$, Masato Suzuki ${ }^{3}$, Keigo Shibayama ${ }^{5}$ and Tetsuya Yagi ${ }^{1,2^{*}}$ (D)

\begin{abstract}
Background: To clarify the molecular epidemiology of carbapenem-resistant Enterobacter cloacae complex (CREC) and the risk factors for acquisition of carbapenemase-producing E. cloacae complex (CPEC).

Methods: Using clinical CREC isolates detected in a Japanese university hospital over 4 years, carbapenemase production was screened with phenotypic methods. Carbapenemase genes were analysed by PCR and sequencing. Molecular epidemiological analyses were conducted with repetitive extragenic palindromic (REP)-PCR and multilocus sequence typing (MLST). CRECs were identified to the subspecies level by hsp60 sequencing. Wholegenome sequencing of plasmids was conducted. A case-control study was performed to identify risk factors for acquisition of CPEC among patients with CREC.

Results: Thirty-nine CRECs including 20 CPECs carrying bla $a_{1 M P-1}$ were identified. Patients with CPEC had longer hospital stay before detection (26.5 days vs. 12 days, $p=0.008$ ), a urinary catheter (odds ratio [OR], 5.36; 95\% confidence interval [Cl], 1.14-30.9; $p=0.023)$, or intubation (OR, 7.53; $95 \% \mathrm{Cl}, 1.47-53.8 ; p=0.008)$ compared to patients without CPEC. Four genetically closely related CPEC clusters were observed, which showed that three of four CPEC clusters corresponded to E. asburiae (ST 53), E. hormaechei subsp. steigerwaltii (ST 113 and ST 1047) and E. cloacae subsp. cloacae (ST 513) by MLST and hsp60 sequencing. Seven representative plasmids shared structures with class I integron containing bla $a_{\mathrm{IMP}-1}$ and IncHI2A replicon type.

Conclusions: A longer hospital stay, presence of a urinary catheter, and intubation are risk factors for CPEC acquisition. In addition to horizontal transmission of genetically indistinguishable CPECS, IncHI2A plasmid carrying bla $a_{\mathrm{IMP}-1}$ appeared to be transferred among genetically different ECs.
\end{abstract}

Keywords: Molecular epidemiology, Carbapenem-resistant Enterobacteriaceae, Carbapenemase-producing Enterobacteriaceae, Enterobacter cloacae complex, $\beta$-Lactamase, Carbapenemase, Repetitive extragenic palindromic polymerase chain reaction, Multilocus sequence typing, Whole-genome sequencing, Plasmid

\footnotetext{
* Correspondence: tyagi@med.nagoya-u.ac.jp

'Department of Infectious Diseases, Nagoya University Hospital, 65 Tsurumai,

Nagoya, Aichi 466-0065, Japan

${ }^{2}$ Department of Infectious Diseases, Nagoya University Graduate School of

Medicine, Nagoya, Aichi, Japan

Full list of author information is available at the end of the article
}

(c) The Author(s). 2019 Open Access This article is distributed under the terms of the Creative Commons Attribution 4.0 International License (http://creativecommons.org/licenses/by/4.0/), which permits unrestricted use, distribution, and reproduction in any medium, provided you give appropriate credit to the original author(s) and the source, provide a link to the Creative Commons license, and indicate if changes were made. The Creative Commons Public Domain Dedication waiver (http://creativecommons.org/publicdomain/zero/1.0/) applies to the data made available in this article, unless otherwise stated. 


\section{Background}

The emergence of carbapenem-resistant Enterobacteriaceae (CRE), especially carbapenemase-producing Enterobacteriaceae (CPE), has been increasing worldwide and is a clinical and public health threat [1]. Since September 2014, CRE infectious diseases have been involved in Category V Infectious Diseases identified by the Act on Prevention of Infectious Diseases and Medical Care for Patients Suffering Infectious Diseases in Japan. The National Epidemiological Surveillance of Infectious Diseases and Japan Nosocomial Infections Surveillance (JANIS) by the Ministry of Health, Labor and Welfare of Japan have been implemented for CRE using the criteria of either meropenem-minimum inhibitory concentration (MIC) $\geq 2 \mu \mathrm{g} / \mathrm{mL}$ or cefmetazole-MIC $\geq 64 \mu \mathrm{g} / \mathrm{mL}$, in addition to imipenem-MIC $\geq 2 \mu \mathrm{g} / \mathrm{mL}$. The annual report from JANIS 2017 showed that the prevalence of CRE is relatively low (0.27\%). About half of CRE involves Enterobacter species (mainly Enterobacter cloacae complex (EC)). IMP-type $\beta$-lactamase is the most common carbapenemase in Japan [2,3]. The latest results of surveillance of Japanese CRE in 2017 revealed that 239 CPE strains (28\%) were detected, and 227 CPE strains carried $b l a_{\mathrm{IMP}}$, of which EC was the most common (74 strains). The IMP genotype was assessed in some strains, and IMP-1 (44\%) and IMP-6 (56\%) were detected [4].

CRE has been analysed predominantly in Klebsiella pneumoniae (KP) because of the initial global spread of CRE of KPC-type $\beta$-lactamase-producing KP strains. Most CRE research has focused on frequently detected species such as KP and Escherichia coli [5]. Among CRE, CPE bacteremia has a fourfold higher mortality rate within 14 days comparing to non-CPE CRE bacteremia [6]. It has been suggested that CPE is more likely to spread than non$\mathrm{CPE}$, and it is listed as one of the important research themes for infection control [7]. Recent meta-analyses of the clinical epidemiology of CRE showed that CREC is the second most common species among the studies that focused on a single species of the Enterobacteriaceae family [8]. In Japan as well, CREC is the second major species of CRE, and molecular characterization of CREC clinical isolates has been reported [9-11]. Further understanding of the molecular epidemiology of CREC and investigation of carbapenemase gene-carrying plasmids, which is the most important resistance mechanism of transmission, are needed to prevent the spread of CREC.

This study aimed to clarify the molecular epidemiology of CPEC isolates and their plasmids carrying carbapenemase genes detected in Nagoya University Hospital (NUH) and to analyse the risk factors for CPEC acquisition compared with CREC without carbapenemase production.

\section{Methods}

\section{Study design and population}

This was a single-centre, retrospective, observational study of hospitalized patients with positive cultures of CREC from April 1, 2012 to March 31, 2016 at NUH, a 1,035-bed tertiary acute care hospital in Japan. The first CREC isolate from a patient at NUH during the study period that met the CRE surveillance definition in Japan was included [3]. A case-control study was conducted to identify risk factors for acquisition of CPEC between patients who acquired CPEC and those who acquired nonCPEC.

\section{Data collection}

Patient information was retrieved from patient electronic medical records. The parameters included demographics, background conditions and comorbidities, recent health care-associated exposure (such as stays in health care facilities), invasive procedures, the presence of indwelling devices, exposure to antimicrobials within 3 months prior to isolation of CREC, and the clinical outcome. Infectious clinical diagnosis was determined according to the information present in medical charts recorded by the attending doctor. The patients were determined to be colonisers if they did not have any signs or symptoms of infection based on information in their medical charts.

\section{Microbiological methods}

Primary identification of bacterial species and antimicrobial susceptibilities was performed using an automated identification and susceptibility testing system (MicroScan WalkAway; Beckman Coulter, Brea, CA, USA) according to Clinical and Laboratory Standards Institute guidelines (document M100-S22), with dry plates (Beckman Coulter) in each case. EC isolates that showed either meropenem-MIC $\geq 2 \mu \mathrm{g} /$ $\mathrm{mL}$ or cefmetazole-MIC $\geq 64 \mu \mathrm{g} / \mathrm{mL}$, in addition to imipenem-MIC $\geq 2 \mu \mathrm{g} / \mathrm{mL}$, according to the definition by the Act on Prevention of Infectious Diseases and Medical Care for Patients Suffering Infectious Diseases for CRE in Japan, were included [3].

\section{Screening of carbapenemase and sequencing of the carbapenemase gene}

CRECs were screened to detect carbapenemase production using disc synergy tests, specific inhibitors, and the modified carbapenemase inactivation method (mCIM) [12]. All mCIM-positive CRECs were screened for the presence of $b l a_{\mathrm{IMP}-1}, b l a_{\mathrm{IMP}-2}, b l a_{\mathrm{NDM}-1}, b l a_{\mathrm{VIM}-2}$, and $b l a_{\mathrm{KPC}}$ genes using PCR with primers as previously described [13-15]. When an isolate tested positive for the carbapenemase gene, its PCR product was sequenced at a commercial laboratory (Eurofin Genomics, Tokyo, 
Japan) and assembled with Sequencher DNA sequence analysis software (Gene Codes, Ann Arbor, MI, USA). Using its consensus sequence, the type of IMP was determined with BLAST (https://blast.ncbi.nlm.nih.gov/ Blast.cgi).

\section{Repetitive extragenic palindromic polymerase chain reaction (REP-PCR)}

DNA was isolated with an UltraClean Microbial DNA isolation kit (MoBio, San Diego, CA, USA) and used for experiments with the Enterobacter spp. fingerprinting kit (bioMerieux Japan, Tokyo, Japan) per the manufacturer's procedure. PCR products were separated by electrophoresis using microfluidic lab-on-a-chip (Agilent Bioanalyzer 2100; Agilent, Santa Clara, CA, USA). Results were analysed using DiversiLab (bioMerieux Japan) on-line software employing the Pearson correlation method, which places more emphasis on the presence or absence of bands than on their intensity. ECs with a similarity of fingerprinting less than $95 \%$ were considered genetically different, and isolates with a similarity of $>98 \%$ were considered indistinguishable [16]. Isolates with a similarity between these values were judged manually using the pattern overlay option in the software. Isolates that were indistinguishable by DiversiLab testing belonged to clusters, and horizontal transmission was defined when an indistinguishable CREC was detected in different patients.

\section{hsp60 PCR and sequencing}

Amplification of $h s p 60$ was accomplished using previously described primers and conditions [17] with DNA extracted with the Cica Geneus DNA Extraction Reagent (Kanto Chemical, Tokyo, Japan). DNA sequencing was performed at a commercial laboratory (Eurofin Genomics) and assembled with Sequencher DNA sequence analysis software (Gene Codes). Based on the neighbour-joining tree of the $h s p 60$ sequences, detailed bacterial names were determined from 12 genetic clusters and an unstable sequence crowd using ClustalW (http://clustalw.ddbj.nig.ac.jp/). Reference strains and type strains were used for neighbourjoining tree of the $h s p 60$ sequences, and the genotypes were determined according to Hoffmann et al [17]. To identify members of E. cloacae complex to subspecies level, species and subspecies were referred to Chavda et al based on $h s p 60$ cluster result [18].

\section{Multilocus sequence typing (MLST)}

DNA was isolated with Cica Geneus DNA Extraction Reagent (Kanto Chemical). Seven housekeeping genes were amplified using primer sets according to the method previously reported $[18,19]$. DNA sequencing was performed at a commercial laboratory (Eurofin
Genomics) and assembled with Sequencher DNA sequence analysis software (Gene Codes). Using its consensus sequence, the sequence type (ST) was determined with the Enterobacter cloacae locus/sequence definitions database (https://pubmlst.org/bigsdb?db=pubmlst_ecloa cae_seqdef). If the sequence did not match the existing ST, new alleles and MLST profiles were registered in the Enterobacter cloacae locus/sequence definitions database (https://pubmlst.org/bigsdb?db=pubmlst_ecloa cae_seqdef).

\section{Whole-genome sequencing}

Plasmids carrying $b l a_{\mathrm{IMP}-1}$ from seven representative isolates were subjected to whole-genome sequencing analysis on a MiniSeq system (Illumina, San Diego, CA, USA), and MinION nanopore sequencer (Oxford Nanopore Technologies, Oxford, UK) using the SQKRBK004 kit and R9.4 flowcells to obtain complete sequences of plasmids carrying the $b l a_{\mathrm{IMP}-1}$ gene. De novo assembly was performed with Unicycler [20] or Miniasm [21], error correction using Illumina reads with Unicycler or CLC Genomics Workbench v9.5.3 (QIAGEN, Hilden, Germany), and coding sequence (CDS) annotation with the PATRIC server (https:// www.patricbrc.org). Linear comparison of $b l a_{\mathrm{IMP}-1}$-carrying plasmid sequences was performed with BLAST and visualized with Easyfig (http://mjsull.github.io/ Easyfig/). The $b l a_{\mathrm{IMP}-1}$ gene, other antimicrobial resistance genes, type IV secretion system-associated genes for conjugation detected by the T346Hunter server [22], and mobile gene elements detected from CDS annotations were indicated.

\section{Statistical analysis}

All analyses were performed using EZR [23]. The association of categorical variables with CREC patients was performed using Fisher's exact test. For continuous data, Mann-Whitney tests were applied appropriately. Statistical significance was considered when the $p$-value was less than 0.05 .

\section{Results}

A total of 39 patients with non-duplicate CREC isolates were identified during the study period. Twenty CPECs among 39 CRECs were revealed with mCIM, and $b l a_{\text {IMP-1 }}$ was identified in all CPEC isolates with negative PCR results for $b l a_{\mathrm{IMP}-2}, b l a_{\mathrm{NDM}-1}, b l a_{\mathrm{VIM}-2}$, and $b l a_{\mathrm{KPC}}$. The annual incidence of the CPEC cases among CREC cases is shown in Fig. 1. The number of CREC cases was six in the first year and ten to eleven cases per year in the subsequent 3 years. About half of CREC was CPEC every year during the study period.

Baseline characteristics and prior health care exposure of the patients with CPEC and non-CPEC are shown in 


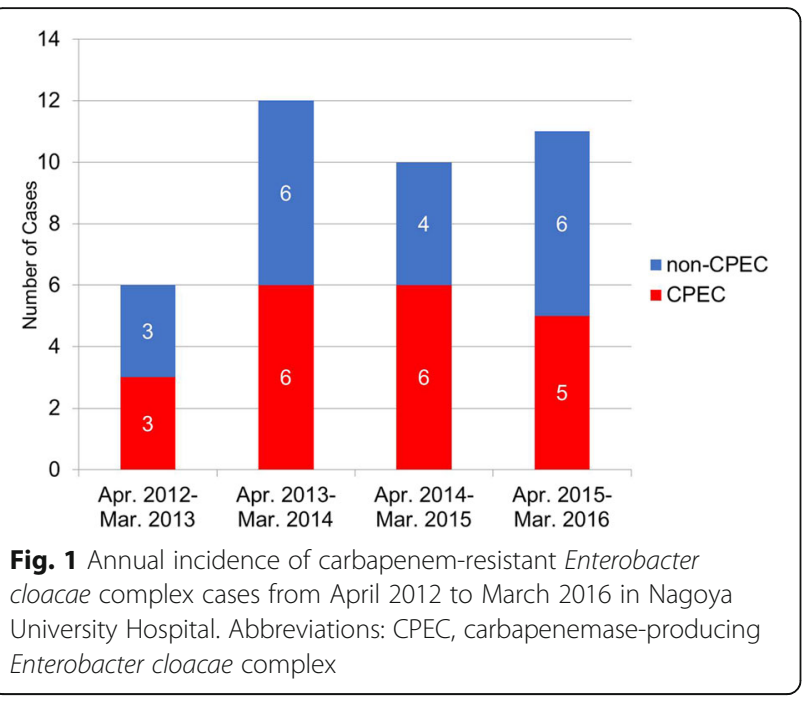

Table 1. CREC isolates were detected most commonly in patients aged 65-79 years old. Almost all individuals had at least one underlying comorbid condition, and approximately $75 \%$ had a history of surgery. Patients with CPEC were more likely to have had a longer hospital stay ( 26.5 days vs. 12 days, $p=0.008$ ), to have had a urinary catheter (odds ratio [OR], 5.36; 95\% confidence interval $[\mathrm{CI}], 1.14-30.9 ; p=0.023)$, and to have been intubated (OR, 7.53; 95\% CI, 1.47-53.8; $p=0.008$ ) before a positive culture result.

The distributions of the culture source, infection types, and outcome of CREC cases are shown in Table 2. CRECs were commonly isolated from nonsterile samples $(34 / 39 ; 87.2 \%)$, and CPECs were detected more frequently from sputum than nonCPECs (OR, 4.40; 95\% CI, 0.94-25.0; $p=0.048$ ). About a half of CREC cases, including five cases (5/ $39 ; 12.8 \%)$ detected from sterile sites, were considered the cause of infection $(21 / 39 ; 53.8 \%)$, and pneumonia $(7 / 39 ; 17.9 \%)$ and peritonitis $(6 / 39 ; 15.4 \%)$ were the most common.

About half of CREC patients $(17 / 39 ; 43.6 \%)$ required a stay in the intensive care unit within 7 days after CREC was cultured, but no significant difference was found between CPEC and non-CPEC cases. Regardless of the culture source, the mortality rate within 30 days or during hospitalization did not differ significantly between CPEC cases and non-CPEC cases.

A molecular epidemiological study with REP-PCR, hsp60, and MLST was performed on all CREC isolates (Fig. 2). REP-PCR differentiated 27 unique genotypes including four CPEC clusters (isolates No. 11-13, No. 15-19, No. 28-29, and No. 30-34) with a similarity cut-off $>95 \%$. Three of four genetically similar CPEC clusters consisted of isolates detected in different years or from patients without an apparent epidemiological relationship. hsp60 sequencing analysis revealed 10 of the 12 genotypes described so far.

Isolates belonging to cluster VIII $(=E$. hormaechei subsp. steigerwaltii) were the most common, accounting for 33.3\% [13] of all isolates, followed by those belonging to cluster I (= E. asburiae) $(9 / 39 ; 23.1 \%)$. Only one isolate (2.6\%) was not classified in Hoffmann cluster. Cluster VII (= E. hormaechei subsp. hormaechei), cluster $\mathrm{X}(=E$. nimipressuralis $)$ and cluster XII $(=E$. cloacae subsp. dissolvens) were not identified in the isolates of this study.

On the other hand, MLST analysis identified 31 unique STs, including 11 novel STs (ST 1043 to ST 1053). Isolates belonging to ST 113 were the most common, accounting for $17.9 \%$ (seven) of all isolates, followed by ST 53 (6/39, 15.4\%). ST 113 and ST 1047 were different in only one of seven loci with seven single nucleotide polymorphisms. Other STs were different in at least three of seven loci.

Both MLST and $h s p 60$ sequencing resulted in a similar clustering pattern as REP-PCR and showed four CPEC clusters that corresponded to cluster I $(=E$. asburiae, ST 53), cluster VIII (=E. hormaechei subsp. steigerwaltii, ST 113 and ST 1047) and cluster XI (=E. cloacae subsp. cloacae, ST 513).

With reference to the phylogenetic tree, wholegenome sequencing was performed with seven representative strains (isolates No. 6, 11, 20, 28, 30, 32, and 35), and analysis of their plasmids was carried out (Table 3).

Six plasmids except for pNUH14_ECL028_1 had almost identical conjugation elements, mobile elements, and a class 1 integron containing bla IMP-1, aac(6')-IIc, and sul1 (Fig. 3). pNUH14_ECL028_1 also had a class 1 integron containing $b l a_{\mathrm{IMP}-1}$, sul1, and aac (6')-1a instead of aac (6')-IIc, and did not have identical conjugation elements and mobile elements compared with the other six plasmids. The insertion sequence ISKpn 7 was located upstream of a class 1 integron, and the insertion sequence IS1 was located downstream of the integron in five plasmids (pNUH12_ECL006_1, pNUH13_ECL030_1, pNUH15_ ECL035_1, pNUH15_ECL032_1 and pNUH15_ECL020_ 1). All seven plasmids carry heavy metal resistance genes for mercury, arsenic and tellurite. All plasmids except for pNUH15_ECL011_1 carry HipBA toxin/antitoxin system.

The data have been deposited with links to BioProject accession number PRJDB7521 in the DDBJ BioProject database. BioSample metadata are available in the DDBJ BioSample database under accession numbers SAMD00143514 to SAMD00143520. The sequence data of plasmids are available in the DDBJ/ EMBL/GenBank databases under the accession numbers AP019382 to AP019388. 
Table 1 Baseline characteristics and health care exposure of carbapenem-resistant Enterobacter cloacae complex cases

\begin{tabular}{|c|c|c|c|c|c|c|}
\hline \multirow{3}{*}{ Baseline characteristics } & \multicolumn{4}{|c|}{ CREC $(n=39)$} & \multirow[t]{2}{*}{ OR $(95 \% \mathrm{Cl})$} & \multirow[t]{2}{*}{$p$} \\
\hline & \multicolumn{2}{|c|}{ CPEC $(n=20)$} & \multicolumn{2}{|c|}{ Non-CPEC $(n=19)$} & & \\
\hline & \multirow{2}{*}{\multicolumn{2}{|c|}{8}} & \multirow{2}{*}{\multicolumn{2}{|c|}{7}} & & \\
\hline Female sex & & & & & \multirow[t]{2}{*}{$1.14(0.26-5.05)$} & 1 \\
\hline Age (years), median (range) & 65 & $(0-82)$ & 71 & $(0-89)$ & & 0.112 \\
\hline \multicolumn{6}{|l|}{ Age group (years) } & \multirow[t]{6}{*}{0.431} \\
\hline $0-18$ & 3 & & 1 & & & \\
\hline $19-49$ & 3 & & 1 & & & \\
\hline $50-64$ & 4 & & 2 & & & \\
\hline $65-79$ & 8 & & 13 & & & \\
\hline$\geqq 80$ & 1 & & 2 & & & \\
\hline Days from admission to positive culture (days), median (range) & 26.5 & $(1-352)$ & 12 & $(0-52)$ & & 0.008 \\
\hline Underlying conditions & 20 & & 17 & & & 0.231 \\
\hline History of surgery & 17 & & 12 & & $3.20(0.58-23.2)$ & 0.155 \\
\hline Any malignancy & 6 & & 9 & & $0.49(0.10-2.12)$ & 0.333 \\
\hline Chronic renal insufficiency & 4 & & 4 & & $0.93(0.15-6.04)$ & 0.939 \\
\hline Neurological disorder & 5 & & 3 & & $1.75(0.28-13.3)$ & 0.695 \\
\hline Congestive heart failure & 3 & & 4 & & $0.67(0.08-4.67)$ & 0.695 \\
\hline Diabetes & 3 & & 3 & & $0.94(0.11-8.11)$ & 1 \\
\hline Transplant recipient & 3 & & 1 & & $3.09(0.22-176)$ & 0.605 \\
\hline Cirrhosis & 1 & & 1 & & $0.95(0.01-78.4)$ & 1 \\
\hline Decubitus or pressure ulcer & 0 & & 1 & & & 0.487 \\
\hline Chronic lung disease & 1 & & 0 & & & 1 \\
\hline Liver failure & 1 & & 0 & & & 1 \\
\hline Urinary tract problems or abnormalities & 0 & & 1 & & & 0.487 \\
\hline Myocardial infarction & 1 & & 0 & & & 1 \\
\hline HIV positive & 0 & & 1 & & & 0.487 \\
\hline \multicolumn{7}{|l|}{ Health care exposure } \\
\hline Administration of antibiotics within 30 days & 18 & & 16 & & $1.67(0.17-22.3)$ & 0.661 \\
\hline Acute care hospitalization within 3 months & 10 & & 13 & & $0.47(0.10-2.03)$ & 0.333 \\
\hline Indwelling devices (2 calendar days prior to culture) & 18 & & 12 & & $5.03(0.78-57.7)$ & 0.065 \\
\hline Central venous catheter & 12 & & 7 & & $2.50(0.60-11.4)$ & 0.205 \\
\hline Nasogastric tube & 11 & & 7 & & $2.06(0.49-9.17)$ & 0.341 \\
\hline Drainage tube & 9 & & 8 & & $1.12(0.27-4.80)$ & 1 \\
\hline Intraperitoneal drainage tube & 6 & & 6 & & $0.93(0.19-4.50)$ & 1 \\
\hline Chest drainage tube & 4 & & 1 & & $4.34(0.38-233)$ & 0.342 \\
\hline Percutaneous transhepatic biliary drainage tube & 3 & & 1 & & $3.09(0.22-176)$ & 0.605 \\
\hline Endoscopic nasobiliary drainage tube & 1 & & 2 & & $0.46(0.007-9.51)$ & 0.605 \\
\hline Urinary catheter & 12 & & 4 & & $5.36(1.14-30.9)$ & 0.023 \\
\hline Intubation (include tracheostomy) & 12 & & 3 & & $7.53(1.47-53.8)$ & 0.008 \\
\hline Haemodialysis & 2 & & 4 & & $0.43(0.03-3.46)$ & 0.407 \\
\hline
\end{tabular}

Note. OR Odds ratio, CI Confidence interval, CREC Carbapenem-resistant Enterobacter cloacae complex, CPEC Carbapenemase-producing Enterobacter cloacae complex 
Table 2 Culture source, clinical diagnosis, and outcome among carbapenem-resistant Enterobacter cloacae complex cases

\begin{tabular}{|c|c|c|c|c|}
\hline & \multicolumn{2}{|l|}{ CREC $(n=39)$} & \multirow[t]{2}{*}{ OR $(95 \% \mathrm{Cl})$} & \multirow[t]{2}{*}{$p$} \\
\hline & CPEC $(n=20)$ & Non-CPEC $(n=19)$ & & \\
\hline \multicolumn{5}{|l|}{ Culture source } \\
\hline Sterile site & 2 & 3 & & \\
\hline Blood & 1 & 2 & $0.46(0.007-9.51)$ & 0.605 \\
\hline Abscess & 1 & 0 & & 1 \\
\hline Vascular graft & 0 & 1 & & 0.487 \\
\hline Non-sterile site & 18 & 16 & & \\
\hline Sputum & 11 & 4 & $4.40(0.94-25.0)$ & 0.048 \\
\hline Peritoneal fluid (not punctured) & 2 & 4 & $0.43(0.03-3.46)$ & 0.407 \\
\hline Urine & 1 & 3 & $0.29(0.05-4.02)$ & 0.342 \\
\hline Bile & 1 & 2 & $0.46(0.007-9.51)$ & 0.605 \\
\hline Oral swab & 0 & 1 & & 0.487 \\
\hline Stool & 2 & 3 & $0.60(0.04-5.96)$ & 0.661 \\
\hline \multicolumn{5}{|l|}{ Clinical diagnosis } \\
\hline Colonisation & 11 & 7 & $2.06(0.49-9.17)$ & 0.341 \\
\hline Infection & 9 & 12 & & \\
\hline Pneumonia & 5 & 2 & $2.76(0.38-33.1)$ & 0.407 \\
\hline Peritonitis & 1 & $5^{\mathrm{a}}$ & $0.15(0.003-1.60)$ & 0.092 \\
\hline Pyelonephritis & 0 & 3 & & 0.106 \\
\hline Bacteremia & 1 & $2^{\mathrm{a}}$ & $0.46(0.007-9.51)$ & 0.605 \\
\hline Cholangitis & 1 & 0 & & 1 \\
\hline Vascular graft infection & 0 & 1 & & 0.487 \\
\hline Abscess & 1 & 0 & & 1 \\
\hline \multicolumn{5}{|l|}{ Outcome } \\
\hline Required intensive care unit stay in the 7 days after positive culture & 10 & 7 & $1.67(0.40-7.48)$ & 0.523 \\
\hline Died at the end of the 30-day evaluation & 1 & 2 & $0.46(0.007-9.51)$ & 0.605 \\
\hline Among sterile site positive culture & 0 & 1 & & \\
\hline Among non-sterile site positive culture & 1 & 1 & & \\
\hline Died during hospitalisation & 3 & 2 & $1.48(0.15-19.9)$ & 1 \\
\hline Among sterile site positive culture & 1 & 1 & & \\
\hline Among non-sterile site positive culture & 2 & 1 & & \\
\hline
\end{tabular}

${ }^{\mathrm{a}}$ One case had peritonitis with bacteremia

Note. OR Odds ratio, CI Confidence interval, CREC Carbapenem-resistant Enterobacter cloacae complex, CPEC Carbapenemase-producing Enterobacter cloacae complex

\section{Discussion}

Molecular epidemiological analysis and genetic characterization of CREC and risk factors for CPEC acquisition over 4 years in a Japanese university-affiliated hospital were performed. Among the patients in whom CREC was detected, long-term hospitalization, the presence of an indwelling urinary catheter, and intubation were associated with isolation of CPEC. Comparison of the fingerprinting patterns by REP-PCR with MLST and hsp60-based genotyping showed general concordance except for isolate No. 39. REP-PCR experiments were conducted repeatedly, but the results were same. The reason why only this isolate did not show concordance is unclear. Molecular epidemiological analysis revealed that genetically indistinguishable CPECs were detected from patients without any epidemiological relationship who were hospitalized in different years, suggesting horizontal transmission, whereas non-CPECs were mostly genetically distinguishable. All CPECs carried $b l a_{\mathrm{IMP}-1}$, and interestingly, even genetically distinct CPECs carried highly similar IncHI2A plasmids harboring $b l a_{\mathrm{IMP}-1}$. These results suggest that the spread of CPECs in the hospital was due to complex mechanisms of a combination of clonal and plasmid transmission. Although we 


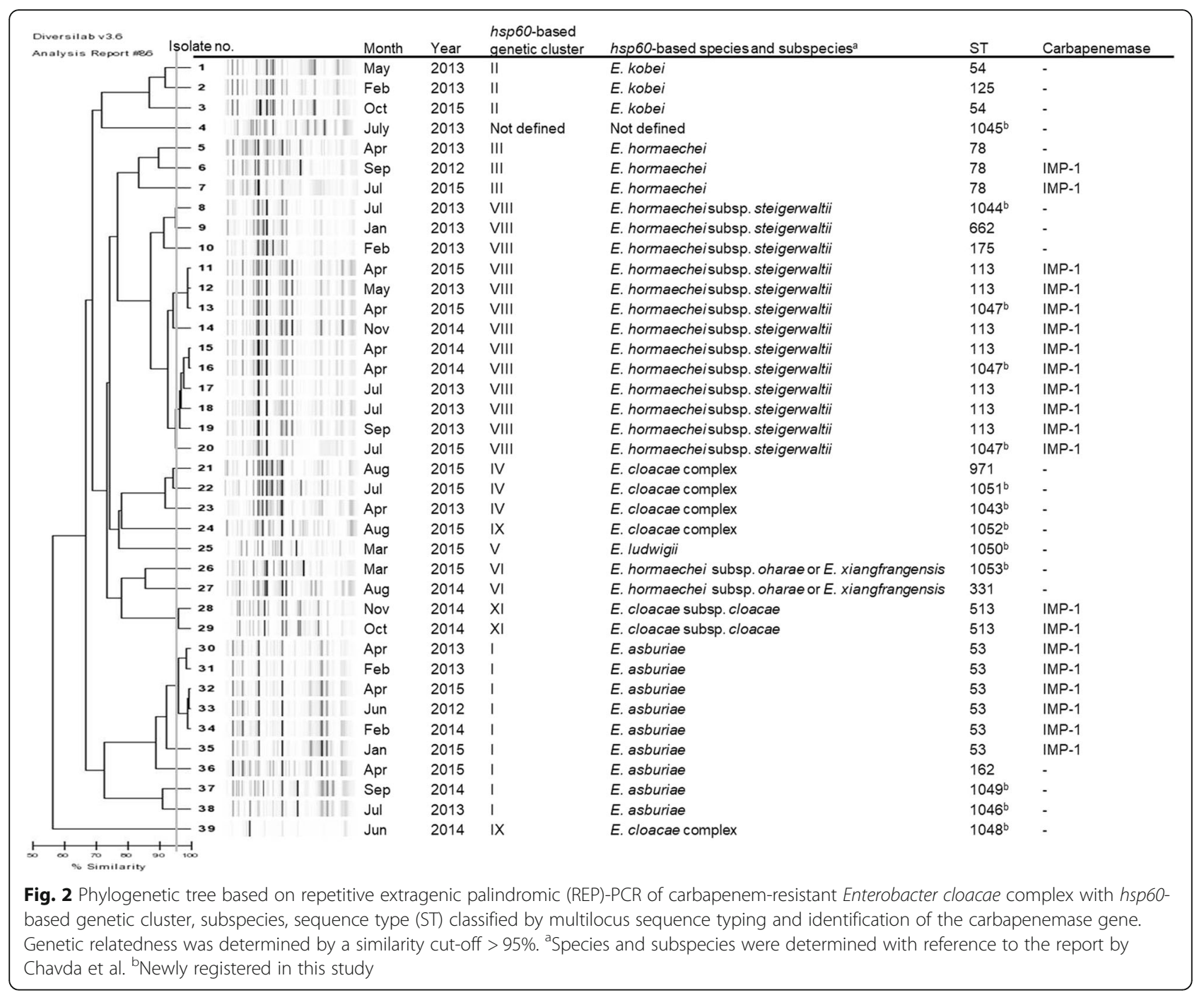

detected no single major clone like ST 235 in K. pneumoniae, among CRECs, CPECs were more likely to be clonally spread. As shown in this study, the clonal characteristics of patients who acquired CPEC compared with patients with non-CPEC, such as prolonged hospitalization or indwelling devices like a urinary tract catheter or intubation, may indicate a greater chance for acquiring of CPECs. Moreover, plasmid analysis of the representative $\mathrm{CPEC}$ isolates revealed common backbone structures, IncHI2A replicon type, class I integron

Table 3 Replicon type, integron type, antimicrobial resistance genes, heavy metal resistance genes and toxin/antitoxin system on the analysed plasmids

\begin{tabular}{|c|c|c|c|c|c|c|c|c|}
\hline \multirow[t]{2}{*}{ Plasmid } & \multirow[t]{2}{*}{ Host } & \multirow{2}{*}{$\begin{array}{l}\text { Plasmid } \\
\text { replicon }\end{array}$} & \multirow{2}{*}{$\begin{array}{l}\text { Integron } \\
\text { type }\end{array}$} & \multirow[t]{2}{*}{ Antimicrobial resistance genes } & \multicolumn{3}{|c|}{ Heavy metal resistance genes } & \multirow{2}{*}{$\begin{array}{l}\text { Toxin/ } \\
\text { antitoxin } \\
\text { system }\end{array}$} \\
\hline & & & & & Mercury & Arsenic & Tellurite & \\
\hline pNUH12_ECL006_1 & 6 & $\operatorname{lncHI2A}$ & Class 1 & $\operatorname{aac}\left(6^{\prime}\right)-\| c, b a_{\mid \mathrm{MP}-1}$, anrB6, sul1, tet(B) & + & + & + & HipBA \\
\hline pNUH15_ECL011_1 & 11 & IncHI2A & Class 1 & $\operatorname{aac}\left(6^{\prime}\right)-\| c, b a_{\mid \mathrm{MP}-1}$, anrB6, sul1, tet(B) & + & + & + & - \\
\hline pNUH15_ECLO20_1 & 20 & IncHI2A & Class 1 & $\operatorname{aac}\left(6^{\prime}\right)-\| c, b l a_{\mid \mathrm{MP}-1}$, anrB6, sul1, tet(B) & + & + & + & HipBA \\
\hline pNUH14_ECLO28_1 & 28 & $\operatorname{lncHI} 2 \mathrm{~A}$ & Class 1 & $\operatorname{aac}\left(6^{\prime}\right)-l a, b l a_{\mathrm{IMP}-1}$, sul1 & + & + & + & HipBA \\
\hline pNUH13_ECLO30_1 & 30 & IncHI2A & Class 1 & $\operatorname{aac}\left(6^{\prime}\right)-\| c$, bla $a_{\mathrm{MP}-1}$, anrB6, sul1, tet(B) & + & + & + & HipBA \\
\hline pNUH15_ECL032_1 & 32 & $\operatorname{lncH} 2 \mathrm{~A}$ & Class 1 & $\operatorname{aac}\left(6^{\prime}\right)-\| c, b l a_{\mathrm{IMP}-1}$, suln, tet $(B)$ & + & + & + & HipBA \\
\hline pNUH15_ECL035_1 & 35 & IncHI2A & Class 1 & $\operatorname{aac}\left(6^{\prime}\right)-\| c, b l a_{\mid \mathrm{MP}-1}$, anrB6, sul1, tet(B) & + & + & + & HipBA \\
\hline
\end{tabular}




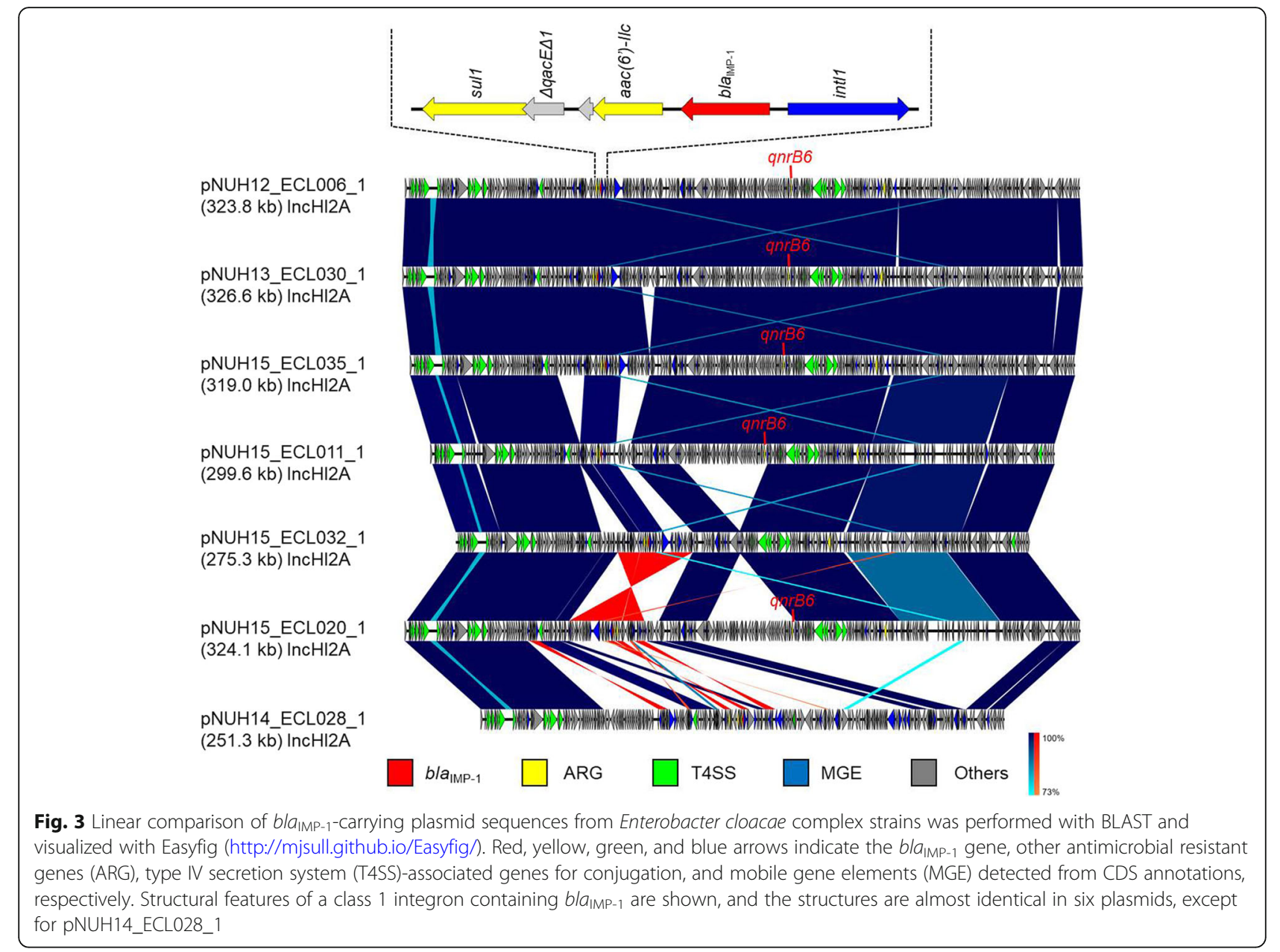

containing $b l a_{\text {IMP-1 }}$ and heavy metal resistance genes and toxin/antitoxin system. Intriguingly, this structure was highly similar to that of pMTY11043 IncHI2 detected from Enterobacter hormaechei (GenBank accession number AP081352.1) reported in Tokyo, Japan [9]. As reported in the study [9], the IncHI2A plasmids may be more likely to have common genes for toxinantitoxin systems and heavy metal resistance, resulting in maintenance of the plasmids, especially in hospital environments [24, 25]. As few studies about CPECs have analysed their plasmids [9, 26, 27], further investigation of molecular epidemiology and plasmid distribution of CPECs at a regional or countrywide level is warranted.

The tendency for clonal transmission of CPECs presented in this study supported the validity of infection control policies focusing on CPE according to the CPE Toolkits published by the Centres for Disease Control and Prevention of England [28, 29]. In our hospital, according to the instructions from the Ministry of Health, Labor and Welfare in Japan, once a CPE not non-CPE CRE, was detected, active surveillance culture of the inpatients in the same ward as the index case was conducted for early detection of asymptomatic carriers. In addition, stringent contact precautions were implemented in both colonised and infected patients to prevent further transmission [30]. However the results of transmission of CPECs between patients hospitalized in different periods and without any apparent epidemiological linkage pose some challenges for infection control in our hospital. Hidden environmental sources such as sink drainage could exist [31, 32], although previous environmental cultures at the responsible intensive care unit and wards found no contamination (data not shown).

This study has some limitations. First, the study was performed at a single centre, and the results cannot be generalized to other institutions. Second, only the E. cloacae complex was targeted in this study among CRE. Whether this result can be applied to other Enterobacteriaceae is unknown.

\section{Conclusions}

Molecular epidemiology and the genetic background of plasmids conferring carbapenem resistance showed 
horizontal transmission of some clones and plasmids with a common backbone of the IncHI2A replicon type and class I integron containing $b l a_{\mathrm{IMP}-1}$. Risk factors for CPEC acquisition are a longer hospital stay and use of indwelling devices, especially intubation and a urinary catheter. Early detection of CPEC and strict infection control measures upon detection including active surveillance culture for asymptomatic carriers are necessary to minimize the transmission of CPECs.

\section{Acknowledgments}

We would like to thank FORTE Science Communications (Tokyo, Japan) for proofreading and editing this manuscript.

\section{Authors' contributions}

TY contributed to the conception and design of this study; NT conceptualised and drafted the manuscript, and performed the microbiological analysis; $\mathrm{AH}, \mathrm{YH}, \mathrm{MI}$ and $\mathrm{MS}$ performed the microbiological analysis and reviewed the manuscript; $\mathrm{AM}, \mathrm{KO}, \mathrm{HM}, \mathrm{YT}$, MS and $\mathrm{KS}$ reviewed the manuscript. All authors read and approved the final manuscript.

\section{Funding}

This work was supported by Grants-in-Aid for Scientific Research Grant Numbers JP 16 K09931 from The Ministry of Education, Culture, Sports, Science and Technology and JP17fk0108307, JP18fk0108061,

JP18fk0108052, and JP18fk0108020 from the Japan Agency for Medical Research and Development.

\section{Availability of data and materials}

All the dataset of this article is available from the corresponding author if reasonably requested.

\section{Ethics approval and consent to participate}

Institutional review boards at NUH approved the study before its initiation (approval number 2017-0444 11811).

\section{Consent for publication}

Not applicable.

\section{Competing interests}

The authors declare that they have no competing interests.

\section{Author details}

'Department of Infectious Diseases, Nagoya University Hospital, 65 Tsurumai, Nagoya, Aichi 466-0065, Japan. ²Department of Infectious Diseases, Nagoya University Graduate School of Medicine, Nagoya, Aichi, Japan. ${ }^{3}$ Antimicrobial Resistance Research Center, National Institute of Infectious Diseases, Tokyo,

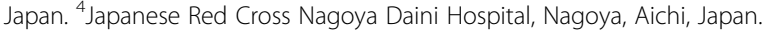
${ }^{5}$ Department of Bacteriology II, National Institute of Infectious Diseases, Tokyo, Japan.

Received: 17 April 2019 Accepted: 15 July 2019

Published online: 24 July 2019

\section{References}

1. Nordmann P, Poirel L. The difficult-to-control spread of carbapenemase producers among Enterobacteriaceae worldwide. Clin Microbiol Infect. 2014;20:821-30.

2. Ministry of Health, Labor and Welfare. Annual Open Report 2016 (All Facilities) Japan Nosocomial Infections Surveillance (JANIS). https://janis. mhlw.go.jp/english/report/open_report/2016/3/1/ken_Open_Report_Eng_2 01600_clsi2012.pdf. Published 2016. Accessed 4 Feb 2019.

3. National Institute of Infectious Diseases. Carbapenem-resistant Enterobacteriaceae Infection, Japan. 2014. Infectious Agents Surveillance Report 2014; 35:281-282. Published 2014. Accessed 4 Feb 2019.

4. National Institute of Infectious Diseases. Surveillance of carbapenemresistant Enterobacteriaceae, 2017. 2018. Infectious Agents Surveillance Report 2018; 39: 162-163. Published 2018. Accessed 4 Feb 2019.
5. Gupta N, Limbago BM, Patel JB, Kallen AJ. Carbapenem-resistant Enterobacteriaceae: epidemiology and prevention. Clin Infect Dis. 2011;53:60-7.

6. Tamma PD, Goodman KE, Harris AD, Tekle T, Roberts A, Taiwo A, et al. Comparing the outcomes of patients with Carbapenemase-producing and non-Carbapenemase-producing Carbapenem-resistant Enterobacteriaceae bacteremia. Clin Infect Dis. 2017;64:257-64.

7. Goodman KE, Simner PJ, Tamma PD, Milstone AM. Infection control implications of heterogeneous resistance mechanisms in carbapenem-resistant Enterobacteriaceae (CRE). Expert Rev Anti-Infect Ther. 2016;14:95-108.

8. van Loon K, Voor In't Holt AF, Vos MC. A systematic review and metaanalyses of the clinical epidemiology of Carbapenem-resistant Enterobacteriaceae. Antimicrob Agents Chemother. 2017;62:e01730-17.

9. Aoki K, Harada S, Yahara K, et al. Molecular characterization of IMP-1producing Enterobacter cloacae Complex isolates in Tokyo. Antimicrob Agents Chemother. 2018;62:e02091-17.

10. Hayakawa K, Miyoshi-Akiyama T, Kirikae T, et al. Molecular and epidemiological characterization of IMP-type metallo-beta-lactamaseproducing Enterobacter cloacae in a large tertiary care hospital in Japan. Antimicrob Agents Chemother. 2014;58:3441-50.

11. Koyano S, Saito R, Nagai $R$, et al. Molecular characterization of carbapenemase-producing clinical isolates of Enterobacteriaceae in a teaching hospital, Japan. J Med Microbiol. 2013;62:446-50.

12. Pierce VM, Simner PJ, Lonsway DR, et al. Modified Carbapenem inactivation method for phenotypic detection of Carbapenemase production among Enterobacteriaceae. J Clin Microbiol. 2017;55:2321-33.

13. Shibata N, Doi Y, Yamane K, et al. PCR typing of genetic determinants for metallo-beta-lactamases and integrases carried by gram-negative bacteria isolated in Japan, with focus on the class 3 integron. J Clin Microbiol. 2003:41:5407-13.

14. Wachino J, Yoshida H, Yamane K, et al. SMB-1, a novel subclass B3 metallobeta-lactamase, associated with ISCR1 and a class 1 integron, from a carbapenem-resistant Serratia marcescens clinical isolate. Antimicrob Agents Chemother. 2011;55:5143-9.

15. Bradford PA, Bratu S, Urban C, et al. Emergence of carbapenemresistant Klebsiella species possessing the class a carbapenemhydrolyzing KPC-2 and inhibitor-resistant TEM-30 beta-lactamases in new York City. Clin Infect Dis. 2004;39:55-60.

16. Fluit AC, Terlingen AM, Andriessen L, Ikawaty R, van Mansfeld R, Top J, et al. Evaluation of the DiversiLab system for detection of hospital outbreaks of infections by different bacterial species. J Clin Microbiol. 2010;48:3979-89.

17. Hoffmann $\mathrm{H}$, Roggenkamp A. Population genetics of the nomenspecies Enterobacter cloacae. Appl Environ Microbiol. 2003;69:5306-18.

18. Chavda KD, Chen L, Fouts DE, Sutton G, Brinkac L, Jenkins SG, et al. Comprehensive genome analysis of Carbapenemase-producing Enterobacter spp.: new insights into phylogeny, population structure, and resistance mechanisms. MBio. 2016. https://doi.org/10.1128/mBio.02093-16.

19. Miyoshi-Akiyama T, Hayakawa K, Ohmagari N, Shimojima M, Kirikae T. Multilocus sequence typing (MLST) for characterization of Enterobacter cloacae. PLoS One. 2013;8:e66358.

20. Wick RR, Judd LM, Gorrie CL, Holt KE. Unicycler: resolving bacterial genome assemblies from short and long sequencing reads. PLoS Comput Biol. 2017;13:e1005595.

21. Li H. Minimap and miniasm: fast mapping and de novo assembly for noisy long sequences. Bioinformatics. 2016:32:2103-10.

22. Martínez-García PM, Ramos C, Rodríguez-Palenzuela P. T346Hunter: a novel web-based tool for the prediction of type III, type IV and type VI secretion systems in bacterial genomes.PLoS One. 2015;10(4):e0119317. https://doi. org/10.1371/journal.pone.0119317. eCollection 2015.

23. Kanda Y. Investigation of the freely available easy-to-use software 'EZR' for medical statistics. Bone Marrow Transplant. 2013:48:452-8.

24. Gullberg E, Albrecht LM, Karlsson C, Sandegren L, Andersson DI. Selection of a multidrug resistance plasmid by sublethal levels of antibiotics and heavy metals. MBio. 2014:5:e01918-4.

25. Mourao J, Marcal S, Ramos P, Campos J, Machado J, Peixe L, et al. Tolerance to multiple metal stressors in emerging non-typhoidal MDR Salmonella serotypes: a relevant role for copper in anaerobic conditions. J Antimicrob Chemother. 2016;71:2147-57.

26. Peirano G, Matsumura $Y$, Adams MD, Bradford P, Motyl M, Chen L, et al. Genomic epidemiology of global Carbapenemase-producing Enterobacter spp., 2008-2014. Emerg Infect Dis. 2018;24:1010-9. 
27. Beyrouthy R, Barets M, Marion E, Dananche C, Dauwalder O, Robin F, et al. Novel Enterobacter lineage as leading cause of nosocomial outbreak involving Carbapenemase-producing strains. Emerg Infect Dis. 2018;24:1505-15.

28. Centers for Disease Control and Prevention. Facility Guidance for Control of Carbapenem-resistant Enterobacteriaceae (CRE) - November 2015 Update CRE Toolkit. 2015. https://www.cdc.gov/hai/organisms/cre/cre-toolkit/. Published 2015. Accessed 4 Feb 2019.

29. Public Health England. Acute trust toolkit for the early detection, management and control of carbapenemase-producing Enterobacteriaceae 2013. https://assets.publishing.service.gov.uk/government/uploads/system/ uploads/attachment_data/file/329227/Acute_trust_toolkit_for_the_early_ detection.pdf. Published 2013. Accessed 4 Feb 2019.

30. Kato D, Morioka H, Tomita Y, lguchi M, Hirabayashi A, Tetsuka N, et al. Active surveillance in response to the identification of a single carbapenemase-producing Escherichia coli at a Japanese university hospital. J Infect Chemother. 2018;24:1013-5.

31. Parkes LO, Hota SS. Sink-related outbreaks and mitigation strategies in healthcare facilities. Curr Infect Dis Rep. 2018;20:42.

32. Kanamori H, Weber DJ, Rutala WA. Healthcare outbreaks associated with a water reservoir and infection prevention strategies. Clin Infect Dis. 2016;62:1423-35.

\section{Publisher's Note}

Springer Nature remains neutral with regard to jurisdictional claims in published maps and institutional affiliations.

Ready to submit your research? Choose BMC and benefit from:

- fast, convenient online submission

- thorough peer review by experienced researchers in your field

- rapid publication on acceptance

- support for research data, including large and complex data types

- gold Open Access which fosters wider collaboration and increased citations

- maximum visibility for your research: over $100 \mathrm{M}$ website views per year

At $\mathrm{BMC}$, research is always in progress.

Learn more biomedcentral.com/submissions 